\title{
Studies on Variability, Correlation and Path Analysis in Red Ripe Chilli Genotypes
}

\author{
Paramjeet Singh Negi* and Akhilesh Sharma \\ Department of Vegetable Science and Floriculture, CSK Himachal Pradesh Krishi \\ Vishvavidyalaya, Palampur, 176062, India \\ *Corresponding author
}

\begin{tabular}{|l|}
\hline Ke y w o r d s \\
Path analysis, \\
Red ripe chilli, \\
Genotypes \\
\hline Article Info \\
\hline Accepted: \\
12 March 2019 \\
Available Online: \\
10 April 2019 \\
\hline
\end{tabular}

A B S T R A C T
The experimental material comprised of 33 genotypes including 'Surajmukhi' as standard check was evaluated in randomized complete block design with three replications during summer-rainy season 2017. The estimates of PCV and GCV were high for dry fruit yield/plant and it also showed high heritability coupled with high genetic advance along with marketable red ripe fruit yield/plant, non- marketable red ripe fruits/plant, dry fruit yield/plant and oleoresin content. Majority of the traits showed high heritability along with moderate estimates of PCV, GCV and genetic advance. Correlation studies revealed that marketable red ripe fruit yield/plant showed positive association with fruit length, fruit girth, fruit width, plant height, average red ripe fruit weight, marketable red ripe fruits/plant, total red ripe fruits/plant, per cent marketable red ripe fruits/plant, average dry fruit weight and dry fruit yield/plant at both the levels. Total red ripe fruits/plant directly contributed maximum toward the marketable red ripe fruit yield/plant followed by per cent marketable red ripe fruits/plant contributed directly to a limited extent at both phenotypic and genotypic levels. Thus, indicating direct selection for these traits as a criterion for yield improvement in chilli.

\section{Introduction}

Chilli is one of the common and remunerative cash crops grown for its green and dry red fruits. It is an indispensable spice due to its pungency, taste, colour and flavor in every house of the tropical world and has its unique place in the diet both as a vegetable and spice crop (Gadaginmath, 1992). Today, India has emerged as the major producer, consumer and exporter of chilli. It is presently grown extensively throughout the country both under rainfed and irrigated conditions in almost all the states and contributes almost one fourth of the world production. In India, green and dry chilli covers an area of 0.032 and 0.084 million hectares with annual production of 3.634 and 2.096 million tonnes, respectively during 2017-18. The initial and cheapest input to enhance the productivity of any crop is to make available high yielding and well adapted varieties by initiating a strong breeding programme. Genetic variability in germplasm decides the level of success in the improvement of such germplasm through selection and provides the possibility to 
improve the yield and quality through strategic breeding programme. Crop improvement with heritable characters, estimation of genetic parameters and their association is of prime importance in breeding (Bozokalfa et al., 2010). The estimate of heritability acts as a predictive instrument in expressing the reliability of phenotypic values and it helps the plant breeders to make selection for a particular character when heritability is high in magnitude (Unche et al., 2008).

Effective improvement in yield may be brought about through selection for yield component characters (Alkuddsi et al., 2013). Favourable associations between desirable attributes will help improvement in a joint manner whereas, unfavourable associations between the desirable attributes under selection may limit genetic advance. Knowledge of correlation alone is often misleading as the correlation observed may not be always true. Simple correlation analysis that relates yield to a single variable may not provide a complete understanding of the importance of each component in determining fruit yield (Okuyama et al., 2004).

Path coefficient analysis allows an effective means of partitioning correlation coefficients into unidirectional pathway and alternate pathways. This analysis permits a critical examination of specific factors that produce a given correlation and can be successfully employed in formulating an effective selection strategy. Selection based on the detailed knowledge of magnitude and direction of association between yield and its attributes is very important in identifying the key characters, which can be exploited for crop improvement through suitable breeding programme. Keeping this in view, the present investigation was carried out to evaluate 33 genotypes for red ripe fruit yield and related horticultural traits.

\section{Materials and Methods}

The experiment was undertaken at the Experimental Farm of Department of Vegetable Science and Floriculture, Chaudhary Sarwan Kumar Himachal Pradesh Krishi Vishvavidyalaya, Palampur during summer 2017. The study location was situated at an elevation of $1,290.8 \mathrm{~m}$ above mean sea level with $32^{\circ} 6^{\prime} \mathrm{N}$ latitude and $76^{\circ} 3^{\prime} \mathrm{E}$ longitude represents the mid-hill zone of Himachal Pradesh with annual precipitation of $2,500 \mathrm{~mm}$. The soil is classified as Alfisolstypic Hapludalf clay having a $\mathrm{pH}$ of 5.7. The experimental material comprised of $27 \mathrm{~F}_{7}$ advanced line derived from eight intervarietal crosses, five entries from AICRP on Vegetable Crops and recommended variety 'Surajmukhi' as the standard check. Seed of 33 genotypes was sown in the nursery bed of size $3 \mathrm{~m} \times 1 \mathrm{~m} \times 15 \mathrm{~cm}$ on $14^{\text {th }}$ March 2017 and were tra planted on $7^{\text {th }}$ May 2017 in randomized complete block design with three replications. Each genotype was planted in two rows of length $2.25 \mathrm{~m}$ consisting of ten plants in each replication with inter and intra row spacing of $45 \mathrm{~cm} \times 45 \mathrm{~cm}$, respectively. The observations were recorded on five competitive plants taken at random over the replications on days to flowering, pedicel length, fruit length, fruit girth, fruit width, leaf length, leaf width, plant height, primary branches per plant, secondary branches per plant, average red ripe fruit weight, marketable red ripe fruits per plant, nonmarketable red ripe fruits per plant, total red ripe fruits per plant, per cent marketable red ripe fruits per plant, red ripe fruit yield per plant, average dry fruit weight, dry fruit yield per plant, oleoresin and capsaicin content.

The genotypic and phenotypic correlations were calculated as per Al- Jibouri et al., (1958) by using analysis of variance and covariance matrix in which total variability had been splitted into replication, genotypes 
and errors. Path coefficient was obtained according to the procedure elaborated by Dewey and Lu (1959).

\section{Results and Discussion}

The knowledge of PCV and GCV is helpful in predicting the amount of variation present in the germplasm which helps in formulating an efficient breeding programme (Table 1). Phenotypic coefficient of variation (PCV) was slightly higher than the genotypic coefficient of variation (GCV) for all the characters studied which indicated that environment also played a considerable role in expression of these characters (Kumar et al., 2014 and Pandit and Adhikari, 2014). PCV and GCV were high for dry fruit yield/ plant indicating sufficient variability ensuring ample scope for improvement of these traits through selection (Pujar et al., 2017; Nahak et al., 2018).

Moderate estimates of PCV and GCV were observed for most of the characters namely, fruit length, secondary branches/plant, average red ripe fruit weight, marketable red ripe fruits/plant, red ripe fruit yield/plant, non- marketable red ripe fruits/plant, total red ripe fruits/plant, average dry fruit weight, oleoresin and capsaicin contents indicating that selection for these traits should be taken up with cautions (Pandit and Adhikary,, 2014; Pujar et al., 2017).

Heritability knowledge influences the choice of breeding procedures to predict gain from selection and to determine the relative importance of genetic effects (Laghari et al., 2010). Estimates of heritability are helpful in studying the inheritance of quantitative characters and also important for planning breeding programmes with desired degree of expected genetic progress. High heritability estimates were observed for 70 per cent of the characters studied namely, fruit length, leaf length, leaf width, plant height, average red ripe fruit weight, marketable red ripe fruits/plant, red ripe fruit yield/plant, nonmarketable red ripe fruits/plant, total red ripe fruits/plant, per cent marketable red ripe fruits/plant, average dry fruit weight, dry fruit yield/plant, oleoresin and capsaicin content indicating greater role of genetic components of variation and lesser influence of environment (Kadwey et al., 2015; Meena et al., 2016).

These characters with high heritability needs to be given due emphasis as they are under genetic control. However, high heritability does not necessarily mean high genetic gain and heritability in alone is insufficient to make predictions for improvement through simple phenotypic selection.

High heritability along with high genetic advance was observed for red ripe fruit yield/plant, non- marketable red ripe fruits/plant, dry fruit yield/plant and oleoresin content (Yatung et al., 2014; Pujar et al., 2017) suggesting the presence of additive gene action. High heritability along with moderate genetic advance was observed for majority of the traits namely, fruit length, leaf length, leaf width, plant height, average red ripe fruit weight, marketable red ripe fruits/plant, total red ripe fruits/plant, average dry fruit weight and capsaicin content indicating the importance of additive and nonadditive gene action (Bijalwan and Naidu 2013, Megharaj et al., 2017).

The success of selection program depends upon the magnitude and direction of association between the trait of interest and yield. After attaining the knowledge of nature and magnitude of genetic variation, it would be important to gather information on association of yield with other characters and among themselves. Genotypic correlation coefficients were higher in magnitude than the corresponding phenotypic ones (Table 2). 
Table.1 Estimates of parameters of variability for various traits in red chilli

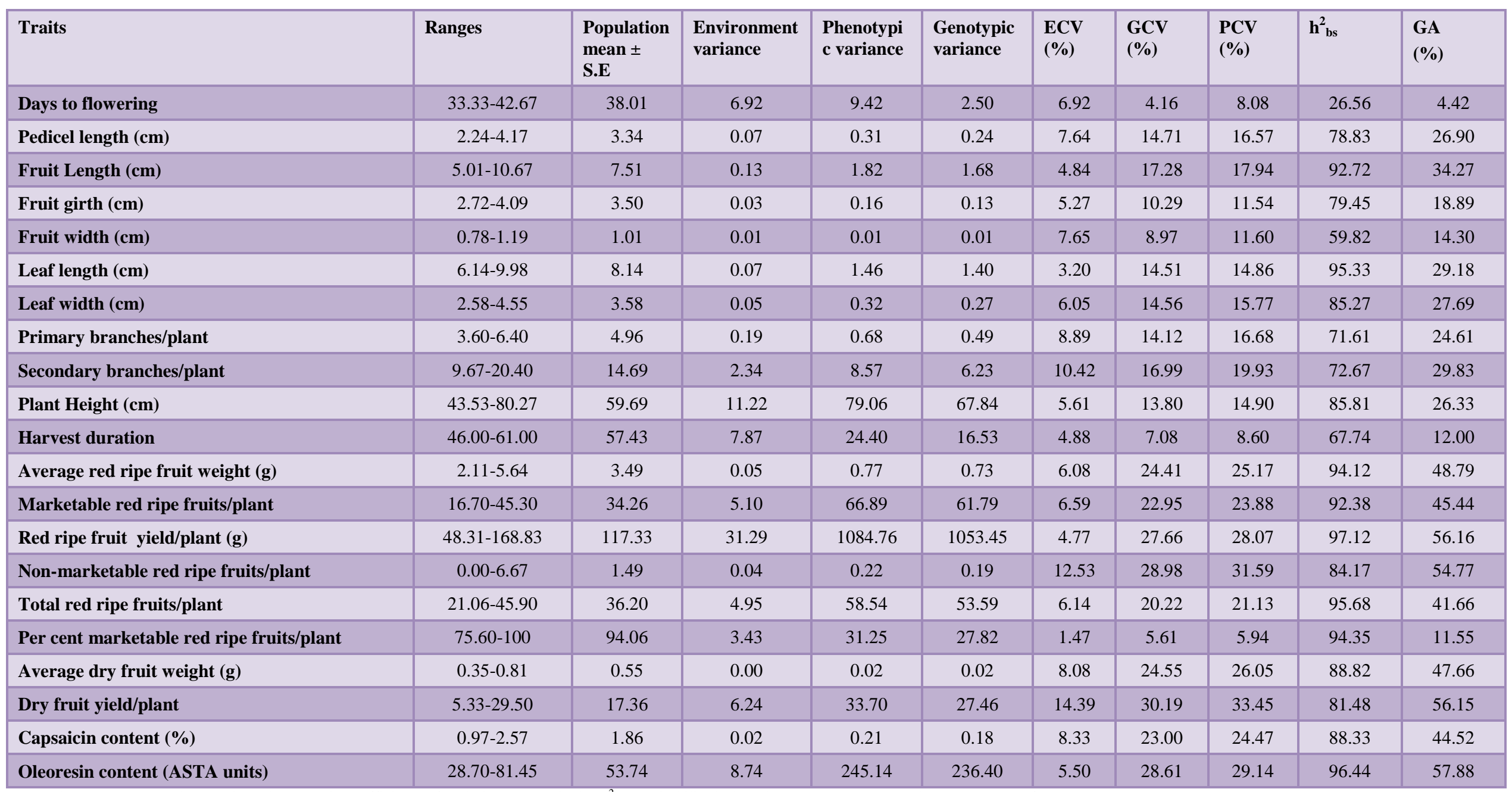

PCV and GCV represent phenotypic and genotypic coefficients of variation, respectively; $\mathrm{h}^{2}$ bs: Heritability in broad sense; GA (\%): Genetic advance (\%) of mean 
Table.2 Estimates of phenotypic and genotypic correlation coefficients for different pair of traits in red chilli

\begin{tabular}{|c|c|c|c|c|c|c|c|c|c|c|c|c|c|c|c|c|c|c|c|c|}
\hline Traits & & $\begin{array}{c}\text { Pedicel } \\
\text { length }(\mathbf{c m})\end{array}$ & $\begin{array}{l}\text { Fruit } \\
\text { Length } \\
\text { (cm) }\end{array}$ & $\begin{array}{l}\text { Fruit girth } \\
\text { (cm) }\end{array}$ & $\begin{array}{c}\text { Fruit width } \\
(\mathrm{cm})\end{array}$ & $\begin{array}{l}\text { Leaf length } \\
(\mathrm{cm})\end{array}$ & $\begin{array}{l}\text { Leaf width } \\
\text { (cm) }\end{array}$ & $\begin{array}{l}\text { Primary } \\
\text { branches/pl } \\
\text { ant }\end{array}$ & $\begin{array}{l}\text { Secondary } \\
\text { lbranches/pl } \\
\text { ant }\end{array}$ & $\begin{array}{c}\text { Plant } \\
\text { Height }(\mathrm{cm})\end{array}$ & $\begin{array}{l}\text { Average } \\
\text { red ripe } \\
\text { fruit weight } \\
\text { (g) }\end{array}$ & $\begin{array}{l}\text { Marketable } \\
\text { red ripe } \\
\text { t fruits/plant }\end{array}$ & $\begin{array}{c}\text { e Non- } \\
\text { marketable } \\
\text { red ripe } \\
\text { fruits/plant }\end{array}$ & $\begin{array}{c}\text { Total red } \\
\text { ripe fruit/ } \\
\text { plant }\end{array}$ & $\begin{array}{c}\text { Percent } \\
\text { marketable } \\
\text { red ripe } \\
\text { fruit }\end{array}$ & $\begin{array}{c}\text { Average } \\
\text { dry fruit } \\
\text { weight (g) }\end{array}$ & $\begin{array}{c}\text { Dry fruit } \\
\text { yield/plant }\end{array}$ & $\begin{array}{c}\text { Capsaicin } \\
\text { content (\%) }\end{array}$ & $\begin{array}{c}\text { Oleoresin } \\
\text { content } \\
\text { (ASTA } \\
\text { units) }\end{array}$ & $\begin{array}{l}\text { Marketable } \\
\text { red ripe } \\
\text { fruit } \\
\text { yield/plant } \\
\text { (g) }\end{array}$ \\
\hline \multirow{2}{*}{ Days to flowering } & $\mathrm{P}$ & 0.179 & 0.084 & -0.018 & 0.092 & -0.041 & 0.181 & -0.040 & -0.052 & -0.036 & -0.152 & -0.184 & -0.039 & $-0.283^{*}$ & -0.110 & 0.012 & $-0.262^{*}$ & $-0.273^{*}$ & -0.036 & $-0.278^{*}$ \\
\hline & $\mathrm{G}$ & 0.184 & 0.065 & -0.087 & 0.145 & -0.046 & $0.295^{*}$ & -0.073 & $-0.262^{*}$ & -0.017 & $-0.288^{*}$ & $-0.405^{*}$ & -0.032 & $-0.567^{*}$ & $-0.251^{*}$ & 0.036 & $-0.585^{*}$ & $-0.511^{*}$ & -0.062 & $-0.558^{*}$ \\
\hline \multirow[t]{2}{*}{ Pedicel length(cm) } & $P$ & & $0.430^{*}$ & -0.036 & -0.114 & 0.006 & -0.119 & -0.019 & $-0.210^{*}$ & $0.328^{*}$ & 0.047 & $-0.202^{*}$ & $0.210^{*}$ & -0.154 & $-0.216^{*}$ & -0.026 & -0.170 & $-0.196^{*}$ & -0.001 & -0.160 \\
\hline & G & & $0.467^{*}$ & -0.055 & $-0.241^{*}$ & 0.015 & -0.185 & -0.014 & $-0.228^{*}$ & $0.406^{*}$ & 0.070 & $-0.229^{*}$ & $0.261^{*}$ & -0.162 & $-0.264^{*}$ & -0.023 & -0.183 & $-0.230^{*}$ & -0.011 & -0.170 \\
\hline \multirow[t]{2}{*}{ Fruit Length $(\mathbf{c m})$} & $P$ & & & $0.303^{*}$ & $0.211^{*}$ & 0.176 & -0.059 & $-0.305^{*}$ & 0.087 & $0.476^{*}$ & $0.465^{*}$ & -0.032 & 0.058 & $0.360^{*}$ & 0.068 & $0.260^{*}$ & 0.161 & -0.162 & $-0.198^{*}$ & $0.352^{*}$ \\
\hline & G & & & $0.300^{*}$ & 0.164 & $0.198^{*}$ & -0.059 & $-0.331^{*}$ & 0.089 & $0.518^{*}$ & $0.501^{*}$ & -0.031 & 0.048 & $0.386^{*}$ & 0.082 & $0.307^{*}$ & $0.220^{*}$ & -0.188 & $-0.204^{*}$ & $0.379^{*}$ \\
\hline \multirow[t]{2}{*}{ Fruit girth $(\mathbf{c m})$} & $\mathrm{P}$ & & & & $0.636^{*}$ & $0.232^{*}$ & $0.204^{*}$ & -0.094 & -0.069 & $0.397^{*}$ & $0.561^{*}$ & -0.135 & -0.111 & $0.396^{*}$ & $0.222^{*}$ & $0.395^{*}$ & 0.190 & $0.244^{*}$ & 0.192 & $0.395^{*}$ \\
\hline & $\mathrm{G}$ & & & & $0.813^{*}$ & $0.283^{*}$ & $0.269^{*}$ & -0.102 & -0.085 & $0.437^{*}$ & $0.648^{*}$ & -0.148 & $-0.205^{*}$ & $0.450^{*}$ & $0.308^{*}$ & $0.480^{*}$ & $0.250^{*}$ & $0.268^{*}$ & $0.221^{*}$ & $0.453^{*}$ \\
\hline \multirow[t]{2}{*}{ Fruit width (cm) } & $P$ & & & & & -0.034 & 0.047 & $-0.201^{*}$ & 0.115 & 0.164 & $0.417^{*}$ & -0.132 & -0.168 & $0.278^{*}$ & 0.157 & $0.373^{*}$ & $0.241^{*}$ & 0.080 & -0.013 & $0.280^{*}$ \\
\hline & G & & & & & -0.002 & 0.151 & $-0.254^{*}$ & 0.170 & 0.176 & $0.566^{*}$ & -0.190 & $-0.257^{*}$ & $0.368^{*}$ & $0.228^{*}$ & $0.508^{*}$ & $0.334^{*}$ & 0.108 & -0.008 & $0.372^{*}$ \\
\hline \multirow[t]{2}{*}{ Leaf length (cm) } & $P$ & & & & & & $0.637^{*}$ & 0.196 & $-0.291^{*}$ & -0.004 & 0.080 & -0.002 & $-0.264^{*}$ & 0.030 & $0.255^{*}$ & 0.014 & 0.099 & $0.440^{*}$ & 0.055 & 0.043 \\
\hline & G & & & & & & $0.677^{*}$ & $0.232^{*}$ & $-0.315^{*}$ & 0.018 & 0.082 & -0.004 & $-0.297^{*}$ & 0.030 & $0.267^{*}$ & 0.015 & 0.115 & $0.472^{*}$ & 0.059 & 0.044 \\
\hline \multirow[t]{2}{*}{ Leaf width(cm) } & $P$ & & & & & & & 0.025 & $-0.372^{*}$ & -0.061 & 0.075 & -0.021 & $-0.366^{*}$ & 0.043 & $0.330^{*}$ & -0.001 & -0.095 & $0.391^{*}$ & -0.086 & 0.060 \\
\hline & G & & & & & & & 0.031 & $-0.399^{*}$ & 0.003 & 0.094 & -0.030 & $-0.407^{*}$ & 0.050 & $0.350^{*}$ & -0.017 & -0.120 & $0.453^{*}$ & -0.111 & 0.068 \\
\hline \multirow{2}{*}{$\begin{array}{l}\text { Primary } \\
\text { branches/plant }\end{array}$} & $P$ & & & & & & & & -0.046 & $-0.268^{*}$ & $-0.454^{*}$ & 0.183 & -0.044 & $-0.235^{*}$ & -0.024 & $-0.339^{*}$ & -0.075 & 0.098 & $0.404^{*}$ & $-0.228^{*}$ \\
\hline & G & & & & & & & & -0.016 & $-0.336^{*}$ & $-0.513^{*}$ & 0.164 & -0.031 & $-0.293^{*}$ & -0.047 & $-0.457^{*}$ & $-0.203^{*}$ & 0.138 & $0.467^{*}$ & $-0.286^{*}$ \\
\hline \multirow{2}{*}{$\begin{array}{l}\text { Secondary } \\
\text { branches/ plant }\end{array}$} & $P$ & & & & & & & & & -0.015 & 0.026 & 0.087 & 0.129 & 0.127 & -0.083 & -0.159 & 0.083 & -0.135 & $-0.264^{*}$ & 0.118 \\
\hline & $\mathrm{G}$ & & & & & & & & & -0.088 & -0.023 & 0.160 & 0.170 & 0.154 & -0.099 & $-0.205^{*}$ & 0.097 & -0.107 & $-0.321^{*}$ & 0.143 \\
\hline \multirow[t]{2}{*}{ Plant Height (cm) } & $P$ & & & & & & & & & & $0.497^{*}$ & -0.090 & 0.092 & $0.390^{*}$ & 0.070 & $0.467^{*}$ & $0.230^{*}$ & 0.113 & 0.039 & $0.381^{*}$ \\
\hline & G & & & & & & & & & & $0.544^{*}$ & -0.092 & 0.098 & $0.432^{*}$ & 0.089 & $0.555^{*}$ & $0.264^{*}$ & 0.107 & 0.047 & $0.423^{*}$ \\
\hline \multirow{2}{*}{$\begin{array}{l}\text { Average red ripe } \\
\text { fruit weight (g) }\end{array}$} & $P$ & & & & & & & & & & & $-0.314^{*}$ & -0.043 & $0.550^{*}$ & $0.272^{*}$ & $0.636^{*}$ & $0.325^{*}$ & 0.171 & -0.130 & $0.545^{*}$ \\
\hline & G & & & & & & & & & & & $-0.286^{*}$ & -0.049 & $0.561^{*}$ & $0.289^{*}$ & $0.682^{*}$ & $0.365^{*}$ & 0.191 & -0.131 & $0.555^{*}$ \\
\hline \multirow{2}{*}{$\begin{array}{l}\text { Marketable red } \\
\text { ripe fruits/ plant }\end{array}$} & $P$ & & & & & & & & & & & & $-0.402^{*}$ & $0.583^{*}$ & $0.537^{*}$ & $-0.333^{*}$ & 0.381 * & 0.014 & 0.000 & $0.594^{*}$ \\
\hline & $\mathrm{G}$ & & & & & & & & & & & & $-0.433^{*}$ & $0.602^{*}$ & $0.560^{*}$ & $-0.375^{*}$ & $0.406^{*}$ & 0.012 & -0.008 & $0.613^{*}$ \\
\hline \multirow{2}{*}{$\begin{array}{l}\text { Non-marketable } \\
\text { red ripe } \\
\text { fruits/plant }\end{array}$} & $P$ & & & & & & & & & & & & & $-0.286^{*}$ & $-0.844^{*}$ & -0.034 & -0.142 & -0.178 & -0.138 & $-0.327^{*}$ \\
\hline & G & & & & & & & & & & & & & $-0.304^{*}$ & $-0.854^{*}$ & -0.009 & -0.153 & $-0.223^{*}$ & -0.148 & $-0.342^{*}$ \\
\hline \multirow{2}{*}{$\begin{array}{l}\text { Total red ripe } \\
\text { fruit/plant }\end{array}$} & $\mathrm{P}$ & & & & & & & & & & & & & & $0.592^{*}$ & $0.258^{*}$ & $0.667^{*}$ & 0.160 & -0.144 & $0.999^{*}$ \\
\hline & G & & & & & & & & & & & & & & $0.610^{*}$ & $0.264^{*}$ & $0.716^{*}$ & 0.184 & -0.148 & $0.999^{*}$ \\
\hline \multirow{2}{*}{$\begin{array}{l}\text { Percent } \\
\text { marketable red } \\
\text { ripe fruit }\end{array}$} & $P$ & & & & & & & & & & & & & & & 0.065 & $0.270^{*}$ & $0.242^{*}$ & 0.058 & $0.624^{*}$ \\
\hline & G & & & & & & & & & & & & & & & 0.047 & $0.290^{*}$ & $0.273^{*}$ & 0.054 & $0.640^{*}$ \\
\hline \multirow{2}{*}{$\begin{array}{l}\text { Average dry fruit } \\
\text { weight (g) }\end{array}$} & $P$ & & & & & & & & & & & & & & & & $0.470^{*}$ & 0.160 & 0.075 & $0.255^{*}$ \\
\hline & G & & & & & & & & & & & & & & & & $0.433^{*}$ & 0.173 & 0.072 & $0.260^{*}$ \\
\hline \multirow{2}{*}{$\begin{array}{l}\text { Dry fruit } \\
\text { yield/plant }\end{array}$} & $P$ & & & & & & & & & & & & & & & & & $0.244^{*}$ & -0.085 & $0.661^{*}$ \\
\hline & G & & & & & & & & & & & & & & & & & $0.290^{*}$ & -0.111 & $0.710^{*}$ \\
\hline \multirow{2}{*}{$\begin{array}{l}\text { Capsaicin content } \\
(\%)\end{array}$} & $P$ & & & & & & & & & & & & & & & & & & 0.136 & 0.167 \\
\hline & $\mathrm{G}$ & & & & & & & & & & & & & & & & & & 0.151 & 0.192 \\
\hline \multirow{2}{*}{$\begin{array}{l}\text { Oleoresin content } \\
\text { (ASTA units) }\end{array}$} & $P$ & & & & & & & & & & & & & & & & & & & -0.135 \\
\hline & $\mathrm{G}$ & & & & & & & & & & & & & & & & & & & -0.139 \\
\hline
\end{tabular}

Residual effect at phenotypic level $(\mathrm{P})=0.0061$, and genotypic level $(\mathrm{G})=0.0029$. Significant at $\mathrm{P} \leq 0.05$; bold values indicate direct effects; $\mathrm{r}$ correlation coefficient with marketable green fruit yield/plant 
Table.3 Estimates of direct and indirect effects of different traits on marketable red fruit yield per plant at phenotypic $(\mathrm{P})$ and genotypic $(\mathrm{G})$ levels in red ripe chilli

\begin{tabular}{|c|c|c|c|c|c|c|c|c|c|c|c|c|c|c|c|c|c|c|c|c|c|}
\hline Traits & & $\begin{array}{c}\text { Days to } \\
\text { flowering }\end{array}$ & $\begin{array}{l}\text { Pedicel } \\
\text { length } \\
(\mathrm{cm})\end{array}$ & $\begin{array}{c}\text { Fruit } \\
\text { Length } \\
\text { (cm) }\end{array}$ & $\begin{array}{l}\text { Fruit } \\
\text { girth } \\
\text { (cm) }\end{array}$ & $\begin{array}{l}\begin{array}{c}\text { Fruit } \\
\text { width } \\
(\mathbf{c m})\end{array} \\
\end{array}$ & $\begin{array}{c}\text { Leaf } \\
\text { length } \\
(\mathrm{cm})\end{array}$ & $\begin{array}{l}\begin{array}{c}\text { Leaf } \\
\text { width } \\
(\mathrm{cm})\end{array} \\
\end{array}$ & $\begin{array}{l}\text { Primary } \\
\text { branches } \\
\text { per plant }\end{array}$ & $\begin{array}{l}\text { Secondary } \\
\text { branches } \\
\text { per plant }\end{array}$ & $\begin{array}{c}\text { Plant } \\
\text { Height } \\
(\mathrm{cm})\end{array}$ & $\begin{array}{c}\text { Average } \\
\text { red ripe } \\
\text { fruit } \\
\text { weight } \\
\text { (g) }\end{array}$ & $\begin{array}{c}\text { Marketable } \\
\text { red ripe } \\
\text { fruits per } \\
\text { plant }\end{array}$ & $\begin{array}{c}\text { Non- } \\
\text { marketable } \\
\text { red ripe } \\
\text { fruits per } \\
\text { plant }\end{array}$ & $\begin{array}{c}\text { Total } \\
\text { red ripe } \\
\text { fruits } \\
\text { per } \\
\text { plant }\end{array}$ & $\begin{array}{c}\text { Percent } \\
\text { marketable } \\
\text { red ripe } \\
\text { fruit }\end{array}$ & $\begin{array}{c}\text { Average } \\
\text { dry fruit } \\
\text { weight } \\
\text { (g) }\end{array}$ & $\begin{array}{c}\text { Dry } \\
\text { fruit } \\
\text { yield } \\
\text { per } \\
\text { plant }\end{array}$ & $\begin{array}{c}\text { Capsaicin } \\
\text { content } \\
(\%)\end{array}$ & $\begin{array}{c}\text { Oleoresin } \\
\text { content } \\
\text { (ASTA } \\
\text { units) }\end{array}$ & $\mathbf{r}$ \\
\hline \multirow[b]{2}{*}{$\begin{array}{l}\text { Days to } \\
\text { flowering }\end{array}$} & $\mathbf{P}$ & -0.001 & 0.000 & 0.000 & 0.000 & 0.000 & 0.000 & 0.000 & 0.000 & 0.000 & 0.000 & 0.000 & 0.000 & 0.001 & -0.278 & -0.002 & 0.000 & 0.001 & 0.000 & 0.000 & $-0.278^{*}$ \\
\hline & G & 0.012 & -0.002 & 0.000 & 0.001 & 0.003 & -0.001 & -0.005 & 0.000 & 0.003 & 0.000 & -0.012 & -0.019 & 0.001 & -0.536 & 0.000 & 0.000 & 0.005 & -0.007 & 0.000 & $-0.5584^{*}$ \\
\hline \multirow{2}{*}{$\begin{array}{l}\text { Pedicel length } \\
(\mathrm{cm})\end{array}$} & $\mathbf{P}$ & 0.000 & 0.001 & 0.000 & 0.000 & 0.000 & 0.000 & 0.000 & 0.000 & 0.000 & 0.000 & 0.000 & 0.000 & -0.007 & -0.151 & -0.004 & 0.000 & 0.001 & 0.000 & 0.000 & -0.160 \\
\hline & G & 0.002 & -0.010 & 0.000 & 0.001 & -0.005 & 0.000 & 0.003 & 0.000 & 0.003 & 0.005 & 0.003 & -0.011 & -0.007 & -0.153 & 0.000 & 0.000 & 0.002 & -0.003 & 0.000 & -0.170 \\
\hline \multirow{2}{*}{$\begin{array}{l}\text { Fruit Length } \\
(\mathrm{cm})\end{array}$} & $\mathbf{P}$ & 0.000 & 0.000 & 0.001 & 0.000 & 0.000 & 0.000 & 0.000 & -0.001 & 0.000 & 0.000 & -0.001 & 0.000 & -0.002 & 0.354 & 0.001 & 0.001 & -0.001 & 0.000 & 0.000 & $0.352^{\circ}$ \\
\hline & G & 0.001 & -0.005 & 0.000 & -0.004 & 0.003 & 0.002 & 0.001 & -0.002 & -0.001 & 0.007 & 0.021 & -0.002 & -0.001 & 0.365 & 0.000 & -0.004 & -0.002 & -0.002 & 0.001 & $0.379^{\circ}$ \\
\hline \multirow{2}{*}{$\begin{array}{l}\text { Fruit girth } \\
(\mathrm{cm})\end{array}$} & $\mathbf{P}$ & 0.000 & 0.000 & 0.000 & 0.000 & -0.001 & 0.000 & 0.000 & 0.000 & 0.000 & 0.000 & -0.002 & 0.000 & 0.004 & 0.389 & 0.004 & 0.002 & -0.001 & 0.000 & 0.000 & $0.395^{\circ}$ \\
\hline & G & -0.001 & 0.001 & 0.000 & -0.013 & 0.015 & 0.003 & -0.005 & -0.001 & 0.001 & 0.006 & 0.028 & -0.007 & 0.006 & 0.426 & 0.000 & -0.006 & -0.002 & 0.003 & -0.001 & $0.453^{\circ}$ \\
\hline \multirow{2}{*}{$\begin{array}{l}\text { Fruit width } \\
(\mathrm{cm})\end{array}$} & $\mathbf{P}$ & 0.000 & 0.000 & 0.000 & 0.000 & -0.001 & 0.000 & 0.000 & 0.000 & 0.000 & 0.000 & -0.001 & 0.000 & 0.005 & 0.274 & 0.003 & 0.002 & -0.001 & 0.000 & 0.000 & $0.280^{\circ}$ \\
\hline & G & 0.002 & 0.002 & 0.000 & -0.010 & 0.019 & 0.000 & -0.003 & -0.001 & -0.002 & 0.002 & 0.024 & -0.009 & 0.007 & 0.348 & 0.000 & -0.006 & -0.003 & 0.001 & 0.000 & $0.372^{\circ}$ \\
\hline \multirow{2}{*}{$\begin{array}{l}\text { Leaf length } \\
(\mathrm{cm})\end{array}$} & $\mathbf{P}$ & 0.000 & 0.000 & 0.000 & 0.000 & 0.000 & 0.000 & 0.001 & 0.000 & 0.000 & 0.000 & 0.000 & 0.000 & 0.009 & 0.030 & 0.004 & 0.000 & 0.000 & 0.000 & 0.000 & 0.043 \\
\hline & G & -0.001 & 0.000 & 0.000 & -0.004 & 0.000 & 0.010 & -0.012 & 0.001 & 0.004 & 0.000 & 0.004 & 0.000 & 0.008 & 0.029 & 0.000 & 0.000 & -0.001 & 0.006 & 0.000 & 0.044 \\
\hline \multirow[b]{2}{*}{$\begin{array}{l}\text { Leaf } \\
\text { width(cm) }\end{array}$} & $\mathbf{P}$ & 0.000 & 0.000 & 0.000 & 0.000 & 0.000 & 0.000 & 0.002 & 0.000 & 0.000 & 0.000 & 0.000 & 0.000 & 0.012 & 0.042 & 0.005 & 0.000 & 0.000 & 0.000 & 0.000 & 0.060 \\
\hline & G & 0.003 & 0.002 & 0.000 & -0.003 & 0.003 & 0.007 & -0.018 & 0.000 & 0.005 & 0.000 & 0.004 & -0.001 & 0.011 & 0.047 & 0.001 & 0.000 & 0.001 & 0.006 & 0.001 & 0.068 \\
\hline \multirow{2}{*}{$\begin{array}{l}\text { Primary } \\
\text { branches per } \\
\text { plant }\end{array}$} & $\mathbf{P}$ & 0.000 & 0.000 & 0.000 & 0.000 & 0.000 & 0.000 & 0.000 & 0.002 & 0.000 & 0.000 & 0.001 & 0.000 & 0.001 & -0.231 & 0.000 & -0.001 & 0.000 & 0.000 & 0.000 & $-0.228^{*}$ \\
\hline & G & -0.001 & 0.000 & 0.000 & 0.001 & -0.005 & 0.002 & -0.001 & 0.005 & 0.000 & -0.004 & -0.022 & 0.008 & 0.001 & -0.277 & 0.000 & 0.005 & 0.002 & 0.002 & -0.003 & $-0.286^{*}$ \\
\hline \multirow{2}{*}{$\begin{array}{l}\text { Secondary } \\
\text { branches per } \\
\text { plant }\end{array}$} & $\mathbf{P}$ & 0.000 & 0.000 & 0.000 & 0.000 & 0.000 & 0.000 & -0.001 & 0.000 & 0.001 & 0.000 & 0.000 & 0.000 & -0.004 & 0.125 & -0.001 & -0.001 & 0.000 & 0.000 & 0.000 & 0.118 \\
\hline & G & -0.003 & 0.002 & 0.000 & 0.001 & 0.003 & -0.003 & 0.007 & 0.000 & -0.013 & -0.001 & -0.001 & 0.008 & -0.005 & 0.146 & 0.000 & 0.002 & -0.001 & -0.001 & 0.002 & 0.143 \\
\hline \multirow[t]{2}{*}{ Plant Height (cm) } & $\mathbf{P}$ & 0.000 & 0.000 & 0.001 & 0.000 & 0.000 & 0.000 & 0.000 & -0.001 & 0.000 & 0.000 & -0.001 & 0.000 & -0.003 & 0.383 & 0.001 & 0.002 & -0.001 & 0.000 & 0.000 & $0.381^{*}$ \\
\hline & G & 0.000 & -0.004 & 0.000 & -0.006 & 0.003 & 0.000 & 0.000 & -0.002 & 0.001 & 0.013 & 0.023 & -0.004 & -0.003 & 0.408 & 0.000 & -0.006 & -0.002 & 0.001 & 0.000 & $0.422^{*}$ \\
\hline \multirow{2}{*}{\begin{tabular}{|l} 
Average red ripe \\
fruit weight (g) \\
\end{tabular}} & $\mathbf{P}$ & 0.000 & 0.000 & 0.001 & 0.000 & 0.000 & 0.000 & 0.000 & -0.001 & 0.000 & 0.000 & -0.003 & 0.000 & 0.001 & 0.541 & 0.004 & 0.003 & -0.001 & 0.000 & 0.000 & 0.545 \\
\hline & G & -0.003 & -0.001 & 0.000 & -0.008 & 0.011 & 0.001 & -0.002 & -0.002 & 0.000 & 0.007 & 0.043 & -0.014 & 0.001 & 0.530 & 0.000 & -0.008 & -0.003 & 0.002 & 0.001 & $0.555^{\circ}$ \\
\hline \multirow{2}{*}{$\begin{array}{l}\text { Marketable red } \\
\text { ripe fruits per } \\
\text { plant }\end{array}$} & $\mathbf{P}$ & 0.000 & 0.000 & 0.000 & 0.000 & 0.000 & 0.000 & 0.000 & 0.000 & 0.000 & 0.000 & 0.001 & 0.000 & 0.013 & 0.574 & 0.009 & -0.001 & -0.002 & 0.000 & 0.000 & $0.594^{*}$ \\
\hline & G & -0.005 & 0.002 & 0.000 & 0.002 & -0.004 & 0.000 & 0.001 & 0.001 & -0.002 & -0.001 & -0.012 & 0.048 & 0.012 & 0.570 & 0.001 & 0.004 & -0.004 & 0.000 & 0.000 & $0.613^{\circ}$ \\
\hline \multirow{2}{*}{$\begin{array}{l}\text { Non-marketable } \\
\text { red ripe fruits per } \\
\text { plant }\end{array}$} & $\mathbf{P}$ & 0.000 & 0.000 & 0.000 & 0.000 & 0.000 & 0.000 & -0.001 & 0.000 & 0.000 & 0.000 & 0.000 & 0.000 & -0.032 & -0.281 & -0.014 & 0.000 & 0.001 & 0.000 & 0.000 & $-0.327^{*}$ \\
\hline & G & 0.000 & -0.003 & 0.000 & 0.003 & -0.005 & -0.003 & 0.008 & 0.000 & -0.002 & 0.001 & -0.002 & -0.021 & -0.028 & -0.288 & -0.001 & 0.000 & 0.001 & -0.003 & 0.001 & $-0.342^{*}$ \\
\hline \multirow{2}{*}{$\begin{array}{l}\text { Total red ripe fruit } \\
\text { per plant }\end{array}$} & $\mathbf{P}$ & 0.000 & 0.000 & 0.000 & 0.000 & 0.000 & 0.000 & 0.000 & 0.000 & 0.000 & 0.000 & -0.002 & 0.000 & 0.009 & 0.983 & 0.010 & 0.001 & -0.003 & 0.000 & 0.000 & $0.999^{*}$ \\
\hline & G & -0.007 & 0.002 & 0.000 & -0.006 & 0.007 & 0.000 & -0.001 & -0.001 & -0.002 & 0.005 & 0.024 & 0.029 & 0.009 & 0.946 & 0.001 & -0.003 & -0.006 & 0.002 & 0.001 & $0.999^{*}$ \\
\hline \multirow{2}{*}{$\begin{array}{l}\text { Percent } \\
\text { marketable red } \\
\text { ripe fruit }\end{array}$} & P & 0.000 & 0.000 & 0.000 & 0.000 & 0.000 & 0.000 & 0.001 & 0.000 & 0.000 & 0.000 & -0.001 & 0.000 & 0.027 & 0.582 & 0.016 & 0.000 & -0.001 & 0.000 & 0.000 & $0.624^{8}$ \\
\hline & G & -0.003 & 0.003 & 0.000 & -0.004 & 0.004 & 0.003 & -0.006 & 0.000 & 0.001 & 0.001 & 0.012 & 0.027 & 0.024 & 0.577 & 0.001 & -0.001 & -0.003 & 0.004 & 0.000 & $0.640^{\circ}$ \\
\hline \multirow{2}{*}{$\begin{array}{l}\text { Average DRY fruit } \\
\text { weight (g) }\end{array}$} & $P$ & 0.000 & 0.000 & 0.000 & 0.000 & 0.000 & 0.000 & 0.000 & -0.001 & 0.000 & 0.000 & -0.002 & 0.000 & 0.001 & 0.254 & 0.001 & 0.004 & -0.002 & 0.000 & 0.000 & $0.255^{*}$ \\
\hline & G & 0.000 & 0.000 & 0.000 & -0.006 & 0.010 & 0.000 & 0.000 & -0.002 & 0.003 & 0.007 & 0.029 & -0.018 & 0.000 & 0.250 & 0.000 & -0.011 & -0.004 & 0.002 & 0.000 & $0.260^{\circ}$ \\
\hline \multirow{2}{*}{$\begin{array}{l}\text { Dry fruit yield per } \\
\text { plant }\end{array}$} & P & 0.000 & 0.000 & 0.000 & 0.000 & 0.000 & 0.000 & 0.000 & 0.000 & 0.000 & 0.000 & -0.001 & 0.000 & 0.005 & 0.656 & 0.004 & 0.002 & -0.004 & 0.000 & 0.000 & $0.661^{*}$ \\
\hline & G & -0.007 & 0.002 & 0.000 & -0.003 & 0.006 & 0.001 & 0.002 & -0.001 & -0.001 & 0.003 & 0.016 & 0.019 & 0.004 & 0.678 & 0.000 & -0.005 & -0.009 & 0.004 & 0.001 & $0.710^{*}$ \\
\hline \multirow{2}{*}{$\begin{array}{l}\text { Capsaicin content } \\
(\%)\end{array}$} & P & 0.000 & 0.000 & 0.000 & 0.000 & 0.000 & 0.000 & 0.001 & 0.000 & 0.000 & 0.000 & -0.001 & 0.000 & 0.006 & 0.157 & 0.004 & 0.001 & -0.001 & 0.000 & 0.000 & 0.166 \\
\hline & G & -0.006 & 0.002 & 0.000 & -0.003 & 0.002 & 0.005 & -0.008 & 0.001 & 0.001 & 0.001 & 0.008 & 0.001 & 0.006 & 0.174 & 0.000 & -0.002 & -0.003 & 0.013 & -0.001 & 0.192 \\
\hline \multirow{2}{*}{$\begin{array}{l}\text { Oleoresin content } \\
\text { (ASTA units) }\end{array}$} & P & 0.000 & 0.000 & 0.000 & 0.000 & 0.000 & 0.000 & 0.000 & 0.001 & 0.000 & 0.000 & 0.000 & 0.000 & 0.005 & -0.142 & 0.001 & 0.000 & 0.000 & 0.000 & 0.000 & -0.134 \\
\hline & G & -0.001 & 0.000 & 0.000 & -0.003 & 0.000 & 0.001 & 0.002 & 0.002 & 0.004 & 0.001 & -0.006 & 0.000 & 0.004 & -0.140 & 0.000 & -0.001 & 0.001 & 0.002 & -0.005 & -0.139 \\
\hline
\end{tabular}

Residual effect at phenotypic level $(\mathrm{P})=0.0061$, and genotypic level $(\mathrm{G})=0.0029$. Significant at $\mathrm{P} \leq 0.05$; bold values indicate direct effects.; correlation coefficient with red fruit yield per plant 
Marketable red ripe fruit yield/plant showed positive and significant correlation with fruit length, fruit girth, fruit width, plant height, average red ripe fruit weight, marketable red ripe fruits/plant, total red ripe fruits/plant, per cent marketable red ripe fruits/plant, average dry fruit weight and dry fruit yield/plant at both genotypic and phenotypic levels. Similarly, dry fruit yield/plant revealed positive association with fruit width, plant height, average red ripe fruit weight, marketable red ripe fruits/plant, total red ripe fruits/plant, per cent marketable red ripe fruits/plant, average dry fruit weight and capsaicin content. Selection on the basis of these traits might leads to higher yield as well as these traits need to be given special focus for the improvement of red and dry fruit yield. Amongst the component traits, a positive and significant association of average red ripe fruit weight was observed with total red ripe fruits/plant, per cent marketable red ripe fruits/plant, average dry fruit weight, dry fruit yield/plant, plant height, fruit girth, fruit width and fruit length while marketable red ripe fruits/plant showed positive correlation with total red ripe fruits/plant, per cent marketable red ripe fruits/plant and dry fruit yield/plant. Similar desirable association was observed for average dry fruit weight with dry fruit yield/plant, fruit girth, fruit width, fruit length and plant height and that of dry fruit yield/plant with capsaicin content, plant height and fruit width.

The end product, yield has often been described as the product of its component traits which show inter-dependence (Wilson 1987). The path coefficient analysis allows partitioning of correlation coefficients into direct and indirect effects of various traits towards dependent variable and it helps in determining the degree of relationship between yield and its component effects. In some cases, the direct effects were observed to be of opposite at corresponding phenotypic and genotypic levels like in days to flowering, pedicel length, fruit girth, fruit width, leaf width, secondary branches/plant and average dry fruit weight. Such a change in direction and magnitude of direct and indirect effects might be due to environmental factors influencing various traits. Therefore, path analysis at phenotypic level may not provide true picture of direct and indirect causes and it would be advisable to understand the contribution of different traits towards the marketable red ripe fruit yield/plant at genotypic level. Marketable red ripe fruit yield/plant taken as dependent variable and other traits as causal variable for correlation studies. Total red ripe fruits/plant directly contributed maximum toward the total association with different traits followed by per cent marketable red ripe fruit and average dry fruit weight at both phenotypic and genotypic levels (Table 3). In addition primary branches/plant, fruit length, red ripe fruit/plant, plant height and capsaicin content both the levels and that of leaf width, secondary branches/plant and oleoresin content at phenotypic level also contributed directly to some extent towards their total association with marketable red ripe fruit yield/plant. Further, partitioning of positive association of fruit length, fruit girth, fruit width, plant height, average red ripe fruit weight, marketable red ripe fruits/plant, total red ripe fruits/plant, per cent marketable red ripe fruits/plant, average dry fruit weight and dry fruit yield/plant was mainly due to the indirect effect through total red ripe fruits per plant (Farhad et al., 2008; Sarkar et al., 2009; Kumar et al., 2012).

\section{References}

Al-Jibouri, H.A., Miller, P.A. and Robinson, H.F. 1958 . Genotypic and environmental variance and covariance in upland cotton crops of inter-specific origin. Agron. J., 50: 
633-636.

Alkuddsi, Y., Patil, S.S., Manjula, S.M. and Pati, B.C. 2013. Correlation studies on yield and its components in inter specific cotton hybrids ( $G$. Hirsutumx G. barbadense) for developing heterotic box. Mole. Plant Breed., 4: 28.

Bijalwan, P. and Naidu, M. 2013. Genetic variability, heritability and genetic advance of growth and yield components of chilli (Capsicum annuum L.) genotypes. Int. J. Sci. Res., 5: 22-29.

Bozokalfa, M.K., Esiyok, D., Ilbi, H. and Kaygisiz, A.T. 2010. Estimates of genetic variability and association studies in quantitative plant traits of Eruca spp. landraces. Genetika, 42: 501-512.

Dewey, D.R. and Lu, K.H. 1959. A correlation and path analysis of components of crested wheat-grass seed production. Agron J., 51: 515518.

Farhad, M., Hasanuzzaman, M., Biswas, B.K., Azad, A.K. and Arifuzzaman, M. 2008. Reliability of yield contributing characters for improving yield potential in chilli (Capsicum annuum L.). Int. J. Sustain. Crop Prod., 3(3): 30-38.

Gadaginmath, N.B. 1992. Studies related to genetics economic and quality traits and exploitation of heterosis in chilli (Capsicum annuum L.). Ph.D, Thesis, Uni. Agric. Sci., Dharwad.

Kadwey, S., Ashwini, D. and Prajapati, S. 2015. Genotypes performance and genetic variability studies in hot chilli (Capsicum annuum L.). Indian J. Agric. Res., 50: 56-60.

Kumar, A., Ahad, I., Kumar, V. and Thakur, S. 2014. Genetic variability and correlation studies for growth and yield characters in chilli (Capsicum annuum L.). J. Spi. \& Arom. Crops, 23: $170-177$.

Kumar, D., Rangare, V.B. and Singh, S.B.D. 2012. Genetic variability, heritability and correlation studies in chilli (Capsicum annuum L.). HortFlora Res Spectrum, 1: 248-252.

Laghari, K.A., Sial, M.A., Afzal, M.A., Mirbahar, A.A., Pirzada, A.J., Dahot, M.U. and Mangrio, S.M. 2010. Heritability studies of yield and yield associated traits in bread wheat. Pak. J. Bot., 42: 111-115.

Meena, M.L., Kumar, N., Meena, J.K. and Rai, T. 2016. Genetic variability, heritability and genetic advance in chilli (Capsicum annuum L.). HortFlora Research Spectrum, 5: 153156.

Megharaj, K.C., Ajjappalavara, P.S., Satish, D. and Tatagar, M.H. 2017. Estimation of genetic variation, path analysis and thrips reaction studies for yield and yield attributing traits in chilli (Capsicum annuum L.). Plant Archives, 17: 353-361.

Nahak, S.C., Nandi, A., Sahu, G.S., Tripathy, P., Dash, S.K., Patnaik, A. and Pradhan, S.R. 2018. Studies on variability, heritability and genetic advance for yield and yield contributing characters in chilli (Capsicum annuum L.). J. Pharmacogn. Phytochem., 7: 25062510.

Okuyama, L.A., Fedeizi, L.C. and Barbosa, J.F. 2004. Correlation and path analysis of yield and its components and plant traits in wheat. Ciência Rural, 6: 1701-1708.

Pandit, M.K. and Adhikary, S. 2014. Variability and heritability estimates in some reproductive characters and yield in chilli (Capsicum annuum L). Int. J. Plant \& Soil Sci., 3: 845-853.

Pujar, U.U., Shantappa, T., Jagadeesha, R.C., 
Gasti, V.D. and Sandhyarani, N. 2017. Genetic variability, heritablity, correlation and path analysis in chilli (Capsicum annuum L.). Int. J. Pure App. Biosci., 5: 579-586.

Sarkar, S., Murmu, D., Chattopadhyay, A. and Hazra, P. 2009. Genetic variability, correlation and path analysis of some morphological characters in chilli. J. Crop Weed 5: $162-166$.

Unche, P.B., Misal, M.B., Borgaonkar, S.B., Godhawale, G.V., Chavan, B.D. and Sawant, D.R.2008. Genetic variability studies in sweet sorghum (Sorghum bicolor L. Moench). Int. J. Plant Sci., 3: 16-18.

Wilson, D.R. 1987. New approaches to understanding the growth and yield of pea crops. In Peas: Management for quality. Agron. Soc. N. Z., 6: 23-28.

Yatung, T., Dubey, K.R., Singh, V., Upadhyay, G. and Pandey, A.K. 2014. Selection parameters for fruit yield and related traits in chilli (Capsicum annuит L.). Bangl. J. Bot., 43: 283291.

\section{How to cite this article:}

Paramjeet Singh Negi and Akhilesh Sharma. 2019. Studies on Variability, Correlation and Path Analysis in Red Ripe Chilli Genotypes. Int.J.Curr.Microbiol.App.Sci. 8(04): 1604-1612. doi: https://doi.org/10.20546/ijcmas.2019.804.186 